\title{
Cholecystokinin gene is expressed in bovine duodenal tissue
}

\author{
AH Suominen, DR Glimm, JJ Kennelly \\ Department of agricultural, food and nutritional science, University of Alberta, Edmonton, T6G 2P5, Canada
}

The neuropeptide cholecystokinin (CCK) is one of the major regulators of bile excretion and pancreatic enzyme secretion. The rat CCK gene was cloned and sequenced by Deschenes et al (1984, Proc NAS, 81 , 726-730) and subsequently CCK gene expression has been demonstrated in rat and mouse duodenal and brain tissue as well as in some human cell lines. Although there is convincing evidence that CCK also has a physiological role as a regulator of satiety in monogastrics, the role of CCK in the control of feed intake in ruminants has not yet been established. In this study, we report the use molecular biology techniques to demonstrate CCK gene expression in the bovine duodenum.

Total RNA and poly ( $\left.\mathrm{A}^{+}\right)$RNA was isolated from both rat and cow duodenal scrapings by the guanidinium-cesium chloride method and oligo $d(T)$ cellulose chromatography as described by Glimm et al (1992, J Dairy Sci, 75, 2687-2705). A rat CCK cDNA (JE Dixon, Purdue University, West Lafayette, IN, USA) was subcloned into pBluescript $(\mathbb{B}$ II SK (Stratagene). A radiolabelled (32P-CTP) CCK antisense RNA probe was generated using T3 polymerase (Promega). The CCK probe was used in a Northern hybridization assay to detect CCK mRNA in total and poly $\left(A^{+}\right)$ samples. RNA depleted of polyadenylated RNA (poly (A-)) served as negative control. Autoradiographic signal were analyzed using an imaging densitometer (BioRad GS-670).

The results of the Northern hybridization assay demonstrated that the CCK gene is expressed in bovine duodenal mucosa cells. The length of the CCK message (approx $800 \mathrm{nt}$ ) was the same in both bovine and rat duodenum which is consistent with previously reported CCK mRNA sizes. The abundance of CCK mRNA in bovine mucosa was much lower than in rat mucosa, so that the signal of the bovine poly A+RNA (about $1 \%$ yield of total RNA) is comparable with the signal obtained from the total RNA in the rat. The difference in duodenal CCK mRNA abundance between species may reflect the relative importance of gut derived $\mathrm{CCK}$ in ruminants versus monogastrics ; however, differences in metabolic rate between species may also account for the differences. Although the level of expression was lower in bovine duodenum than that observed in rat duodenum, the results reveal that bovine mucosa cells likely synthesize functional CCK. This finding may indicate that CCK plays a similar role in controlling feed intake in ruminants as in monogastrics.

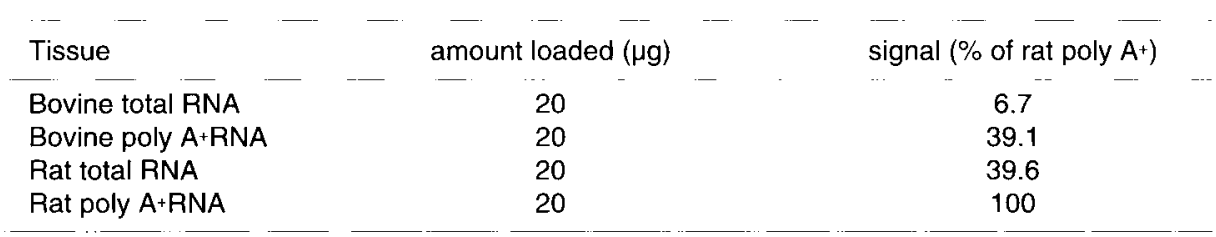

